\title{
Fractional repetitive control of nanopositioning stages for high-speed scanning using low-pass FIR variable fractional delay filter
}

\author{
Linlin Li, Zaozao Chen, Sumeet S. Aphale, Senior Member, IEEE, and LiMin Zhu*, Member, IEEE
}

\begin{abstract}
The Repetitive Control (RC), capable of tracking periodic trajectories and rejecting periodic disturbances, is a promising technique to control the nanopositioning stages for high-speed raster scanning. In digital implementation of the RC scheme, the number of delay points has to be an integer, which implies that the sampling frequency should be an integer multiple of the desired tracking frequency. Clearly, this is a severe limitation on the range of the trajectory frequencies where the RC scheme can effectively be applied. To overcome this limitation, this paper proposes a low-pass FIR variable fractional delay filter as an alternative to the conventional interpolating method employed in conventional fractional RC scheme. This filter combines the interpolating and the low-pass filtering that are employed in the fractional RC and its coefficients are analytically computed as a function of fractional delay, thereby making it suitable for trajectories of all frequencies. The weightedleast-square method is employed to design the low-pass FIR variable fractional delay filter, where the weights are tuned to minimize the approximation errors within the bandwidth-ofinterested. Experimental results are presented to demonstrate the advantages of the proposed method over the conventional RC scheme as well as the interpolating based fractional $\mathrm{RC}$ scheme. These results show that the proposed RC scheme with lowpass FIR variable fractional delay filter improves the tracking performance of the nanopositioner significantly, especially for the trajectories with high-frequency.
\end{abstract}

Index Terms-Nanopositioning stages, high-speed raster scanning, repetitive control, fractional delay filter, nonsynchronized sampling

\section{INTRODUCTION}

Manipulating matter at the nanometer scales was made possible by the invention of the Atomic Force Microscope [1]. Since then, nanotechnology has gained significant momentum and has impacted a wide range of scientific and technological branches [2], [3]. Due to several attractive features such as robustness, repeatability, absence of friction and stiction, ease of integration and simple drive mechanism, piezoelectricallyactuated nanopositioning stages are employed in Atomic Force

This work was supported by the National Natural Science Foundation of China under Grant 51975375, the Binks Trust Visiting Research Fellowship (2018) (University of Aberdeen, UK) awarded to Dr. Sumeet S. Aphale and the SJTU overseas study grant awarded to Linlin Li. The authors would like to thank Mr. Wulin Yan for his assistance with the experiments. (Corresponding author: LiMin Zhu.)

L. Li, Z. Chen and L.M. Zhu are with State Key Laboratory of Mechanical System and Vibration, School of Mechanical Engineering, Shanghai Jiao Tong University, 200240, China.

S. S. Aphale is with the Centre for Applied Dynamics Research, School of Engineering, University of Aberdeen, Aberdeen AB24 3UE, UK.

L.M. Zhu is also with the Shanghai Key Lab of Advanced Manufacturing Environment, Shanghai, China (e-mail: zhulm@sjtu.edu.cn).
Microscopes in order to move the sample to be scanned [4], [5]. The most common used scanning trajectory is a raster pattern - a combination of triangular motion along the fast axis and a ramp / staircase motion along the slow axis. Due to the lightly damped resonance of the mechanical platform and the hysteresis possessed by the piezoelectric actuators used, generating an error-free triangular motion is difficult [6]. This problem is further compounded by rate and amplitude dependent behavior of hysteresis as well as cross-coupling and creep issues. To address this limitation, several open- and closed-loop control techniques have been proposed in literature [5], [7].

Open-loop approaches include model inversion-based input shaping [8], optimal trajectory design [9], non-triangular trajectories [10], [11] and notch-filter-based techniques [12]. However, these techniques lack the wide-band robustness or flexibility that practical control techniques for nanopositioners absolutely require. As a result, closed-loop techniques have garnered a significant research focus. Most of reported closedloop techniques combine a damping loop (to impart damping to the lightly damped resonant mode of the stage which in turn facilitates a wider tracking bandwidth) and a tracking loop (to minimize the effect of hysteresis and other disturbances). As pure integral (I) or proportional and integral (PI) controllers are employed in the tracking loop, these control schemes mainly differ in the damping schemes they adopt. Consequently, the combined damping and tracking control schemes based on Positive Position Feedback [13], Positive Velocity and Position Feedback [13], Positive Acceleration, Velocity and Position Feedback [14], Resonant Controller [15], Integral Resonant Control [16] etc., have been reported till date. In addition to these techniques, Robust Control [17], [18], Fractional Order Control [19], Delayed Position Feedback Control [20], Iterative Control [21]-[23], and Repetitive Control [24] have also shown significant performance improvements.

In high-speed scanning of nanopositioning stages, Iterative Control (including the Iterative Learning Control (ILC) [21], [22] and the Inversion-based Iterative Control (IIC) [23], [25], [26]) and the Repetitive Control (RC), due to their many advantages such as the capability of tracking periodic trajectories, have emerged as promising candidates to further the envelope of high-speed nanopositioning. In comparison, the ILC method requires to reinitialization of its initial value at every iteration period, and the IIC requires several offline training iterations and rather complicated convergence analysis, adding another level of complexity in implementation. From this 
perspective, the RC scheme becomes more attractive, and has delivered significant positioning performance improvements [24], [27], [28]. According to its inherent structure, the RC scheme renders itself ideal for digital implementation, which can be expressed as $1 /\left(z^{N}-1\right)$. This digital RC scheme works on the principle of first dividing a period of the desired periodic trajectory into a number of delay (sample) points and then generating high gains at the harmonics. To guarantee desired control performance and robust stability, normally a low-pass filter and a phase compensator are incorporated into the RC scheme. This standard RC scheme was further enhanced to the Modified RC scheme [29]. Recently, a Dual-Stage RC scheme has also been reported, with significant improvements in tracking accuracy [30]. The main limitation of all these reported $\mathrm{RC}$ scheme variants is the strict requirement of the number of delay points being an integer. In other words, the sampling frequency must be an integer multiple of the frequency of the periodic trajectory to be tracked, i.e., $N=f_{s} / f_{d}$, in which $f_{s}$ and $f_{d}$ denote the sampling frequency and the desired tracking frequency, respectively. This criterion significantly limits the choice of useable scanning frequencies. Although using variable sampling rates can overcome this limitation, the re-modeling and re-design of the control parameters are both time-consuming and complicated.

To address the limitation of having the sampling frequency being an integer multiple of the desired trajectory frequency, three different approaches have been proposed thus far. The first approach is to round-off the fractional delay (due to the sampling frequency not being an integer multiple of the trajectory frequency) to the nearest integer delay. It is clear that this rounding introduces errors and leads to significant performance degradation, especially when high-frequency trajectories are employed [31]. The second approach is employing a frequency adaptive RC schemes [32]. Though feasible in theory, in practice the variable sampling rate significantly increases the real-time implementation complexity and computational burden. The third and widely applied approach is to use a Lagrange interpolating to approximate the fractional delay [31], [33], [34], which becomes the widely used Lagrange Interpolating based Fractional Repetitive Control (LIFRC). In these applications, the Lagrange interpolating to approximate the fractional delay and the low-pass filter to improve the control robustness are applied separately and independently. Due to the poor performance of the equivalent filter at higher frequencies, the performance of the LIFRC degrades when applied to nanopositioning stages for high-frequency scanning. In this case, variable fractional delay filters have emerged as a possible solution to this problem.

Inspired by the design of variable fractional delay filters as reported in [35], [36], this paper proposes the Low-Pass FIR Variable Fractional Delay Filter (LP FIR-VFDF) scheme to handle the rounding issue in the implementation of the RC scheme, thereby enabling an arbitrary choice of the frequency for desired scanning trajectory. This filter combines the Lagrange interpolating to approximate the fractional delay and the low-pass filter to eliminate the influence of the model uncertainty together. The coefficients of LP FIR-VFDF are analytically computed as a function of fractional delay, thereby making it suitable for trajectories of all frequencies. The simulated and experimental results conducted on a custombuilt nanopositioning stage verify the effectiveness of the proposed LP FIR-VFDF based RC scheme.

The rest of this paper is organized as follows. Sec. II provides the design of the LP FIR-VFDF and its application to RC. Its performance is evaluated by comparing it with the commonly used Lagrange interpolating method. Sec. III provides the details of the experimental setup and also lists the used control parameters. Experimental tracking results for the RC, LIFRC as well as the proposed LP FIR-VFDF based RC are presented in Sec. IV for triangular trajectories with a wide range of frequencies, clearly demonstrating the superiority of the proposed scheme. Sec. V concludes the paper.

\section{Controller Design and Performance EVALUATION}

Since the main focus of this work is to design and implement the LP FIR-VFDF in order to improve the performance of the conventional RC scheme under the constraint of synchronized sampling, the design of this filter is first presented here. This will be followed by the application of this filter to the $\mathrm{RC}$ scheme. For sake of comparison, the widely used LIFRC is also briefly introduced.

\section{A. Design of the Low-Pass FIR Variable Fractional Delay Filter}

In order to address the rounding issue of the conventional $\mathrm{RC}$ scheme in high-speed scanning of the nanopositioning stage, the LP FIR-VFDF is introduced to work as both the low-pass filter and the fractional delay filter simultaneously. The ideal frequency response of the LP FIR-VFDF is

$$
H(\omega, d)=A(\omega) e^{-j \omega d}, \quad \omega \in[0, \pi], d \in[0,1]
$$

where $A(\omega)$ is the magnitude response of the filter, $d$ is the fractional delay ranging from 0 to 1 , and the term $e^{-j \omega d}$ denotes the phase response of the filter. Note that as a lowpass filter, the magnitude function $A(\omega)$ in the passband should ideally be 1, attenuate as required (based on the filter order and type) within the transition-band and go to 0 in the stopband. In the passband, the response $H(\omega, d)$ has the expression $e^{-j \omega d}$, which corresponds to the transfer function of $z^{-d}$ in the $z$ domain. The weighted-least-square method as reported in [35], [36], is adopted to design the LP FIR-VFDF approximating its ideal frequency response.

The designed LP FIR-VFDF can then be given by:

$$
F(z, d)=\sum_{n=N_{1}}^{N_{2}} f_{n}(d) z^{-n}
$$

where $N_{1}$ and $N_{2}$ are two integers determining the length of the filter, and the filter coefficients $f_{n}(d)$ are calculated by:

$$
f_{n}(d)=\sum_{k=0}^{K} g(n, k) d^{k}, N_{1} \leq n \leq N_{2}
$$

The parameter $K$ is a positive integer that is related with the approximation performance and $g(n, k)$ is the corresponding 
coefficient with respect to $n$ and $k$. To achieve a good compromise between approximation performance and filter complexity, $K=10$ is chosen in this work. Substituting Eq. (3) into Eq. (2) results in the following expression

$$
F(z, d)=\sum_{n=N_{1}}^{N_{2}} \sum_{k=0}^{K} g(n, k) d^{k} z^{-n}
$$

To optimize the coefficients of the LP FIR-VFDF, we define the parameter matrix $G$ as:

$$
G=\left(\begin{array}{cccc}
g\left(N_{1}, 0\right), & \cdots g\left(N_{1}, k\right), & \cdots & g\left(N_{1}, K\right) \\
\vdots & & \vdots \\
g(n, 0) & \ddots & g(n, K) \\
\vdots & & \vdots \\
g\left(N_{2}, 0\right), & \cdots g\left(N_{2}, k\right), & \cdots & g\left(N_{2}, K\right)
\end{array}\right)
$$

in which $N_{1} \leq n \leq N_{2}, 0 \leq k \leq K$, such that $G$ is of the size $\left(N_{2}-N_{1}+1\right) \times(K+1)$. In this case, the weightedleast-square approximation error $E(G)$ between the frequency response of the designed LP FIR-VFDF and that of the ideal one can be computed as follows.

$$
\begin{array}{r}
E(G)=\sum_{i=0}^{S_{\omega}} \sum_{j=0}^{S_{d}} W\left(\omega_{i}\right)\left|F\left(e^{j w_{i}}, d_{j}\right)-H\left(e^{j w_{i}}, d_{j}\right)\right|^{2} \\
=\sum_{i=0}^{S_{\omega}} \sum_{j=0}^{S_{d}} W\left(\omega_{i}\right)\left[A\left(\omega_{i}\right) \cos \left(\omega_{i} d_{j}\right)\right. \\
\left.-\sum_{n=N_{1}}^{N_{2}} \sum_{k=0}^{K} g(n, k) d_{j}{ }^{k} \cos \left(n \omega_{i}\right)\right]^{2} \\
+\sum_{i=0}^{S_{\omega}} \sum_{j=0}^{S_{d}} W\left(\omega_{i}\right)\left[-A\left(\omega_{i}\right) \sin \left(\omega_{i} d_{j}\right)\right. \\
\left.+\sum_{n=N_{1}}^{N_{2}} \sum_{k=0}^{K} g(n, k) d_{j}{ }^{k} \sin \left(n \omega_{i}\right)\right]^{2}
\end{array}
$$

where $\omega_{i} \in[0, \pi]$ with $\omega_{i}=\frac{i \pi}{S_{\omega}}$, and $d_{j} \in[0,1]$ with $d_{j}=\frac{j}{S_{d}}$. The constants $S_{\omega}$ and $S_{d}$ are the total number of $\omega_{i}$ and $d_{j}$, respectively. The term $W\left(\omega_{i}\right)$ is the weight of the specific frequency $\omega_{i}$. Therefore, defining

$$
\begin{aligned}
E_{c}(G)=\sum_{i=0}^{S_{\omega}} \sum_{j=0}^{S_{d}} W\left(\omega_{i}\right) & {\left[A\left(\omega_{i}\right) \cos \left(\omega_{i} d_{j}\right)\right.} \\
& \left.-\sum_{n=N_{1}}^{N_{2}} \sum_{k=0}^{K} g(n, k) d_{j}{ }^{k} \cos \left(n \omega_{i}\right)\right]^{2}
\end{aligned}
$$

and

$$
\begin{aligned}
E_{s}(G)=\sum_{i=0}^{S_{\omega}} \sum_{j=0}^{S_{d}} W\left(\omega_{i}\right) & {\left[-A\left(\omega_{i}\right) \sin \left(\omega_{i} d_{j}\right)\right.} \\
& \left.+\sum_{n=N_{1}}^{N_{2}} \sum_{k=0}^{K} g(n, k) d_{j}{ }^{k} \sin \left(n \omega_{i}\right)\right]^{2}
\end{aligned}
$$

results in $E(G)=E_{c}(G)+E_{s}(G)$. To further simplify the computations, the following matrices are defined:

$$
\begin{gathered}
R=\left[W^{\frac{1}{2}}\left(\omega_{i}\right) A\left(\omega_{i}\right) \cos \left(d_{j} \omega_{i}\right)\right], \\
I=\left[W^{\frac{1}{2}}\left(\omega_{i}\right) A\left(\omega_{i}\right) \sin \left(d_{j} \omega_{i}\right)\right], \\
P=\left[W^{\frac{1}{2}}\left(\omega_{i}\right) \cos \left(n \omega_{i}\right)\right], \\
Q=\left[W^{\frac{1}{2}}\left(\omega_{i}\right) \sin \left(n \omega_{i}\right)\right],
\end{gathered}
$$

and $D=\left[d_{j}^{k}\right]$. Therefore, the Eqs. (7) and (8) can be rewritten as

$$
E_{c}(G)=\operatorname{tr}\left[\left(R-P G D^{T}\right)^{T}\left(R-P G D^{T}\right)\right]
$$

and

$$
E_{s}(G)=\operatorname{tr}\left[\left(I-Q G D^{T}\right)^{T}\left(I-Q G D^{T}\right)\right]
$$

where the operators $\operatorname{tr}($.$) and T$ are the trace and transpose of the corresponding matrix, respectively. It is evident from Eqs. (13) and (14) that $E(G)$ is a function of $G$. Therefore, the error function $E(G)$ can be minimized if conditions are found such that the partial derivative of the $E(G)$ with respect to $G$ equals 0 . Thus,

$$
\begin{aligned}
& \frac{\partial E(G)}{\partial G}=\frac{\partial E_{c}(G)}{\partial G}+\frac{\partial E_{s}(G)}{\partial G}=0 \\
& =-2 P^{T} R D+2 P^{T} P G D^{T} D-2 Q^{T} I D+2 Q^{T} Q G D^{T} D=0
\end{aligned}
$$

Rearranging terms in Eq. (15), the parameter matrix $G$ is found to be

$G=\left(P^{T} P+Q^{T} Q\right)^{-1}\left(P^{T} R+Q^{T} I\right) D\left(D^{T} D\right)^{-1}$, s.t.K $<S_{d}$

where the existence of $\left(P^{T} P+Q^{T} Q\right)^{-1}$ and $\left(D^{T} D\right)^{-1}$ are proved in the appendices A and B. Consequently, with the obtained parameter matrix $G$, the filters for different values of $d$ can be calculated via Eq. (4).

\section{B. Design of the LP FIR-VFDF based Fractional Repetitive Control}

With the objective of precisely tracking high-frequency periodic trajectories without the constraint of having the sampling frequency be an integer multiple of the trajectory frequency, the LP FIR-VFDF is incorporated into the conventional RC scheme to control nanopositioning stages possessing hysteresis nonlinearity and non-minimum phase dynamics. The overall block diagram of LP FIR-VFDF based Fractional Repetitive Control is shown in Fig. 1. Since the piezo-actuated nanopositioning stages exhibit both linear dynamics and complicated hysteresis nonlinearities, the system model typically adopted is a cascaded structure of the hysteresis model and the linear dynamics transfer function [5]. It is further found that this hysteresis nonlinearity can be modelled as a set of bounded periodic disturbances $d_{h}(k)$ injected to the dynamics, when the system is dedicated to tracking periodic trajectories [29]. Consequently, the piezo-actuated nanopositioning stage is described as the dynamics $P(z)$ cascaded with the 
LP FIR-VFDF based Fractional Repetitive Controller

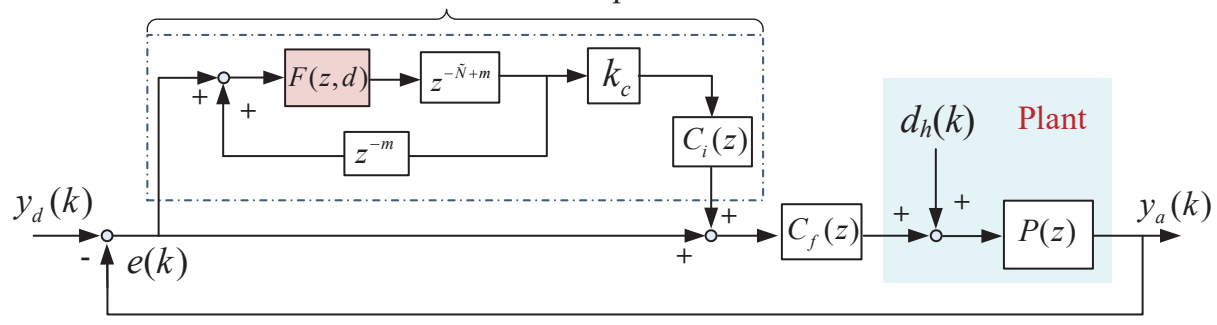

Fig. 1. The block diagram of the control system with LP FIR-VFDF based Fractional Repetitive Control.

bounded disturbances $d_{h}(k)$ resulting from the hysteresis of the piezoelectric actuator. The terms $y_{d}(k), y_{a}(k)$ and $e_{k}$ are the desired input trajectory, the actual output displacement and the corresponding tracking error, respectively. In order to generate the control voltage, the LP FIR-VFDF based Fractional Repetitive Controller is plugged into the baseline feedback control scheme with the tracking controller $C_{f}(z)$. With its ease of implementation, $C_{f}(z)$ is chosen as a simple PI controller whose transfer function is given by $k_{p}+k_{i} \frac{T_{s}}{z-1}$, where $T_{s}$ is the sampling time in this work. The control gains will be tuned via the trial and error method to achieve the best possible tracking performance. The control gain $k_{c}$ is a constant chosen appropriately to guarantee stability of the control system. The block $C_{i}(z)$ is the inversion of the baseline closed-loop system that includes the plant $P(z)$ and the tracking controller $C_{f}(z)$. Thus, $C_{i}(z)$ takes the form:

$$
C_{i}(z)=\left[\frac{P(z) C_{f}(z)}{1+P(z) C_{f}(z)}\right]^{-1}=\left[z^{-r} \frac{B_{s}(z) B_{u}(z)}{A(z)}\right]^{-1}
$$

Due to the non-minimum phase nature of the stage, there exist zeros outside of the unit circle in the $z$-plane, making the direct inversion unrealizable. As expressed in Eq. (17), the zeros are decomposed into two groups. The minimumphase zeros are grouped into $B_{s}(z)$, while the non-minimum phase zeros are grouped into $B_{u}(z)$. The constant $r$ is the relative degree of the transfer function in Eq. (17). To derive a stable inversion, the Zero-phase-tracking-error method [37] is chosen. Consequently, the stable, causal inversion of the baseline closed-loop dynamics obtained can be rewritten as:

$$
C_{i}(z)=z^{-\left(n_{c}+n_{u}\right)} \frac{B_{f}(z) A(z)}{B_{s}(z) \beta^{2}}
$$

The term $B_{f}(z)$ is determined by inverting the coefficients of $B_{u}(z)$. The parameters $n_{u}$ and $n_{c}$ are the order of $B_{u}(z)$ and the compensated order of $C_{i}(z)$ to make it causal, respectively. The constant $\beta$ is given by $\beta=\left|\beta_{0}\right|+\left|\beta_{1}\right|+\left|\beta_{2}\right|+\cdots$, where $\beta_{i}$ are the corresponding coefficients of $B_{u}(z)$. The block $z^{-m}$ is the phase compensator of the inversion, with the order $m=n_{c}+r$.

Since this work focuses on the fractional (non-integer) ratio between the sampling frequency and the trajectory frequency, the number of delay (sample) points in the delay loop is calculated by rounding $N$ to the negative infinity, i.e., $\tilde{N}=\operatorname{floor}(N)$. The residual fractional delay $z^{-d}$ will be compensated by the designed LP FIR-VFDF. It should be noted that, the basic control diagram of the conventional RC is the same as that of the proposed LP FIR-VFDF based Fractional Repetitive Control shown in Fig. 1, except that the $F(z, d)$ is replaced by a conventional low-pass filter and the number of delay points is rounded to an nearest integer value $(\hat{N}=\operatorname{round}(N))$.

\section{Theorem 1. (Ascertaining Stability)}

In order to provide a guideline of the parameters selection of the proposed LP FIR-VFDF based Fractional Repetitive Control scheme, the stability analysis is demonstrated herewith.

As the LP FIR-VFDF based Fractional Repetitive Control is a plugged-in (retrofitted) scheme, the baseline system with PI controller should first be asymptotically stable. Assume this condition is satisfied, which implies that the polynomial $1+$ $P C_{f}=0$ has no roots outside the unit circle in the $z$-plane (the indices of $z$-domain and the fractional delay $d$ are omitted in the stability analysis). With the control scheme shown in Fig. 1 , the characteristic polynomial of the closed-loop system can be derived as

$$
D=\left(1+P C_{f}\right)\left[1-F z^{-\tilde{N}}\left(1-k_{c} z^{m} C_{i} T\right)\right]
$$

where

$$
T=\frac{P C_{f}}{1+P C_{f}}
$$

It is assumed that the first item of Eq. (19) is stable, and consequently the stability of the closed-loop system relies on the second item. According to the Small-Gain Theorem, the system is asymptotically stable, when the following condition is satisfied for all the frequencies.

$$
\left|F z^{-\tilde{N}}\left(1-k_{c} z^{m} C_{i} T\right)\right|<1
$$

Clearly, $|F| \leq 1$ and $\left|z^{-\tilde{N}}\right|=1$. From Eqs. (17) and (18), we have $z^{m} C_{i} T=B_{u} B_{f} / \beta^{2}$. Hence, this inequality can be simplified to

$$
\left|\left(1-k_{c} B_{u} B_{f} / \beta^{2}\right)\right|<1
$$

Since $\beta$ is selected as $\beta=\left|\beta_{0}\right|+\left|\beta_{1}\right|+\left|\beta_{2}\right|+\cdots$ in this work, $\beta^{2} \geq \max \left(B_{u} B_{f}\right)$. As a result, the stability condition of the closed-loop system can thus be stated as

$$
0<k_{c}<2 \text {. }
$$



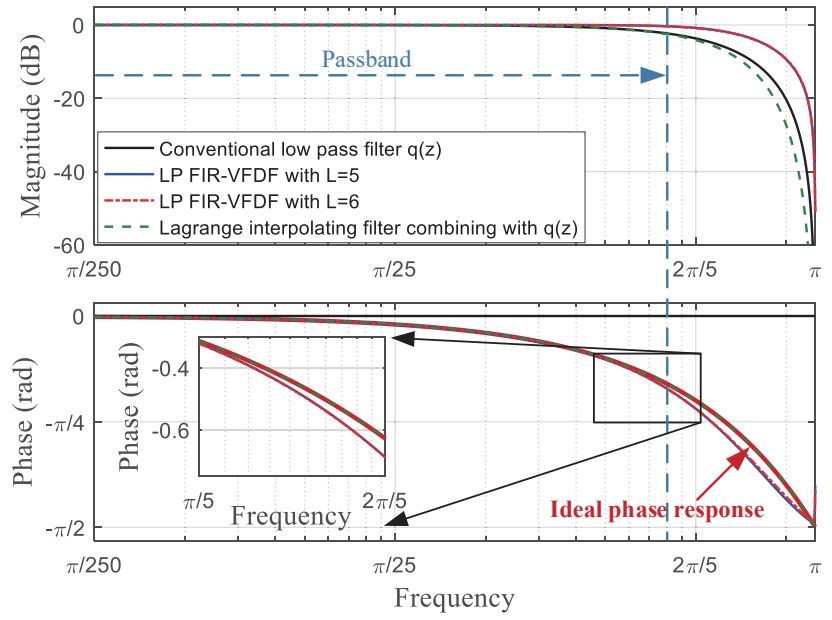

Fig. 2. The comparison of the frequency responses of different filters with $d=0.5$.

\section{Design of Lagrange Interpolating based Fractional Repet- itive Control}

In order to further demonstrate the effectiveness of the LP FIR-VFDF based Fractional Repetitive Control, the commonly employed LIFRC is selected for comparison. In this method, the fractional delay is calculated as:

$$
L(z, d) \approx \sum_{k=0}^{M} A_{k} z^{-k}
$$

with

$$
A_{k}=\prod_{i=0, i \neq k}^{M} \frac{d-i}{k-i}, \quad k=0,1,2 \cdots M
$$

where $M$ is the order of the Lagrange interpolation and is chosen as 3 in most reported works with the tradeoff between the approximation accuracy and computational complexity [31], [33]. The LIFRC is implemented by replacing the $F(z, d)$ in Fig. 1 with $L(z, d)$ cascading with a low-pass filter. Since the effect of the interpolation improves when $d \approx M / 2$ [38], the delay of the LIFRC is approximated in the range $[1,2]$. In this sense, the number of delayed points is then calculated as $\widehat{N}=$ floor $(N)-1$. A full implementation of the LIFRC on a nanopositioner is reported in [31].

\section{Filter performance evaluation}

To evaluate the performance of the LP FIR-VFDF, it is compared with that obtained by (a) the low-pass filter used in the conventional RC and (b) the cascaded two filters (the Lagrange interpolating and the low-pass filter) used in the LIFRC. Since the simplest and optimized zero-phase low-pass filter used in the conventional RC has the form $q(z)=0.25 z^{-1}+0.5+0.25 z$ [23], [25], the low-pass behavior of the LP FIR-VFDF is designed to approximately the same, for a fair comparison. The frequency response of $q(z)$ is shown in Fig. 2 with the solid black line. Following this, the passband, the transition-band and the stopband are chosen as $[0,0.32 \pi),[0.32 \pi, 0.99 \pi)$ and $[0.99 \pi, \pi]$, respectively. $S_{\omega}$ and $S_{d}$ are both chosen as 100 , which implies that the frequency resolution and fractional
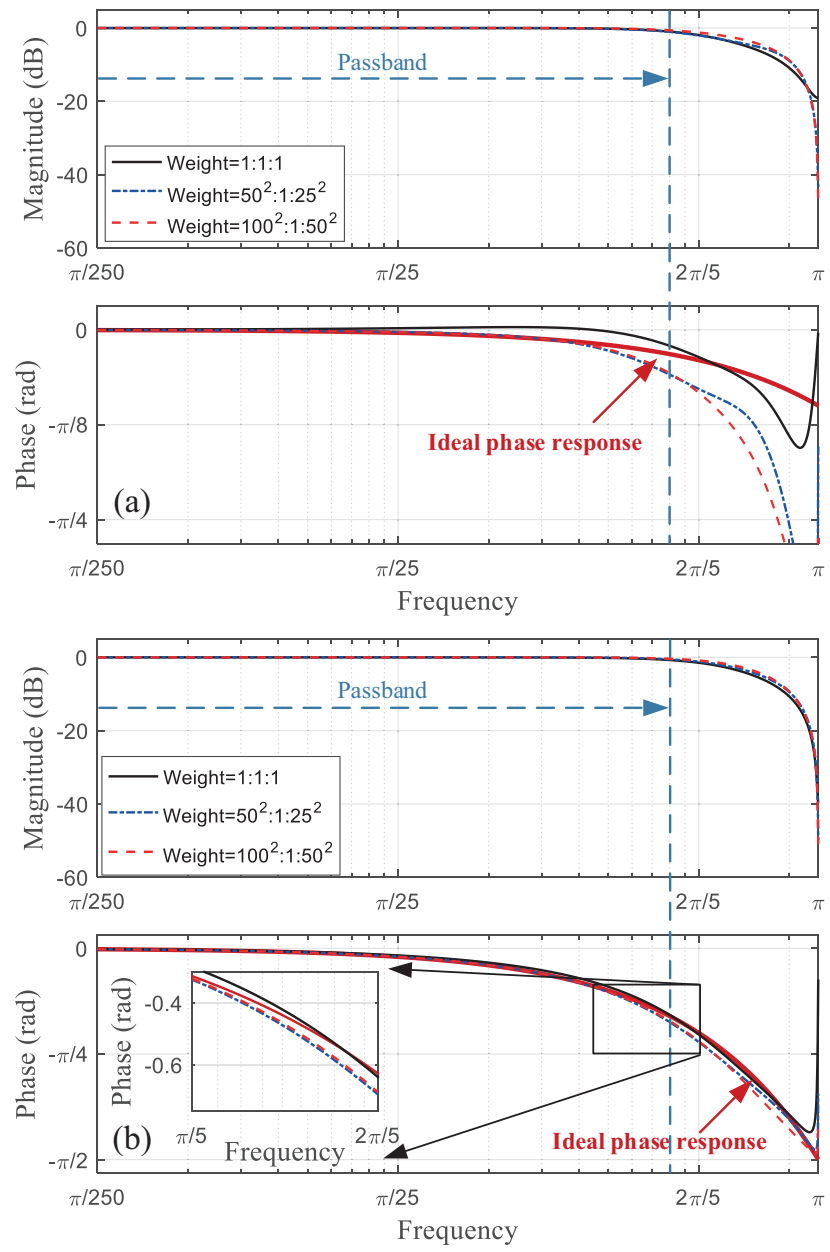

Fig. 3. The frequency responses of the LP FIR-VFDFs with different weights for different values of $d$ : (a) $d=0.1$; (b) $d=0.5$.

delay resolution are $0.01 \pi$ and 0.01 , respectively. The length of the LP FIR-VFDF is selected the same as that of the cascaded filters in the LIFRC, which is $L=N_{2}-N_{1}+1=6$. To determine the filter coefficients, the weights in Eq. (6) have to be initialized. To begin with, the weights are set to 1 for all the frequencies. The parameter $N_{1}$ is selected as -1 to obtain the minimal approximation error. The results for the values of $d=0.1$ and $d=0.5$ are shown in Fig. 3(a) and Fig. 3(b), respectively. It can be seen that although the approximation error is relatively low for $d=0.5$, that of the phase response is quite large for $d=0.1$. Meanwhile, the results in passband and stopband are not satisfied. All these would lead to a severe degradation in tracking performance. Since our focuses are the constant gain and linear phase response in the passband, the weights in the passband, transition-band and the stopband are set as $50^{2}: 1: 25^{2}$ and $100^{2}: 1: 50^{2}$, respectively. The filters obtained with these two weights are also shown in Fig. 3. It is observed that with the adjusted weights, the phase responses are gradually close to the ideal line, and a wider bandwidth is achieved. Consequently, the weights are selected as $100^{2}: 1: 50^{2}$ in this work.

With the weights of $100^{2}: 1: 50^{2}$, the LP FIR-VFDF with $L=5$ is also designed and implemented. The frequency 


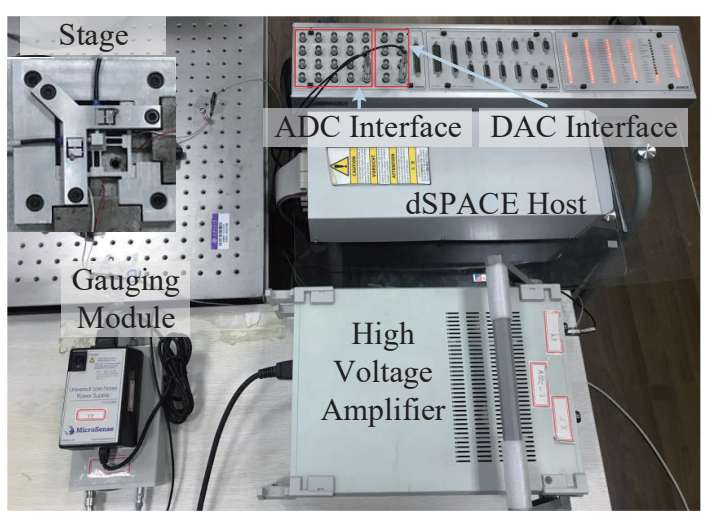

Fig. 4. The experimental setup showing the nanopositioning stage, the dSPACE module, the high-voltage amplifier and the capacitive sensor (gauging module).

responses of the conventional low-pass filter $q(z)$, the cascaded two filters in the LIFRC and LP FIR-VFDF with different values of $L$ are compared in Fig. 2. It can be observed that with the Lagrange interpolation method, the pass-bandwidth is reduced resulting in a degraded tracking of high-frequency trajectories. In contrast, although the phase responses of the LP FIR-VFDFs are worse than that of the equivalent filter in the LIFRC, they are almost the same within the interested passband. More importantly, the LP FIR-VFDFs exhibit wider pass-bandwidth, which would guarantee the tracking precision of the RC in high-speed scanning. It is also useful to note that, with the increase in the filter length, the approximation error can be further reduced. For a fair comparison, the length of the LP FIR-VFDF is set to $L=6$ in the following experiments.

\section{EXPERIMENTAL SYSTEM DESCRIPTION}

\section{A. Experimental setup}

To verify the effectiveness of the proposed LP FIR-VFDF based Fractional Repetitive Control, the experiments are conducted on a two-axis custom-built flexure-hinge nanopositioning stage. The overall experimental setup is shown in Fig. 4. The workspace of the stage is $11.2 \mu \mathrm{m} \times 11.6 \mu \mathrm{m}$. Since the cross-coupling effect between these two scanning axes is less than $0.52 \%$ of the full range, the axes can be treated with the decoupled performance. The Y-axis with its first resonance at $14 \mathrm{kHz}$, is selected for the experiments. The displacement along each axis is measured by a capacitive sensor (Probe 2823 and Gauging Module 8810 from MicroSense, resolution $<1 n m-r m s$ ) via the ADCs (Analog to Digital converters) of the dSPACE. The dSPACE-DS1103 is configured to capture the real-time displacement signals for closed-loop control. The control diagram is established via Matlab/Simulink and downloaded into the dSPACE host, operated via Controldesk. The actuation voltage generated by the dSPACE is fed into the high voltage amplifier, which has an amplification factor of 20 and a range of 0-200 V, via DACs (Digital to Analog converters). The sampling time $T_{s}$ of the dSPACE control system is set to $2 \mu s$ (See [39] for more details).

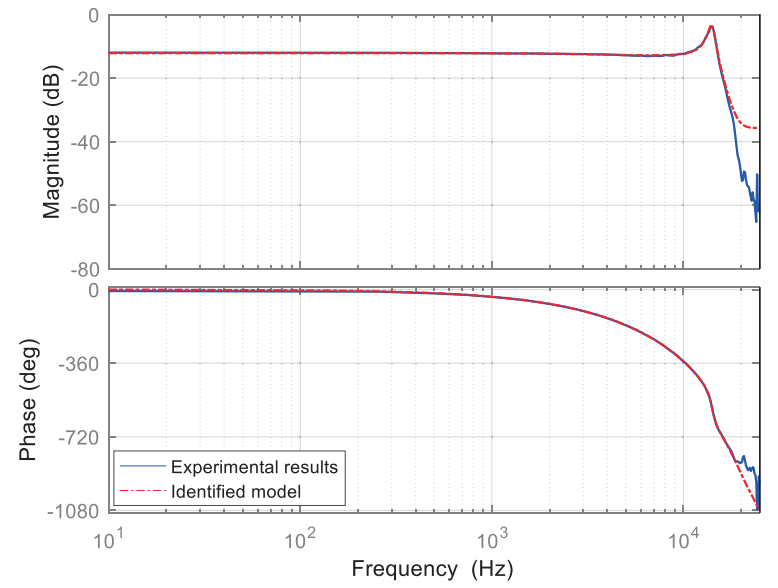

Fig. 5. Measured frequency response of the system (blue) superimposed on that of the identified model (red) showing that the identified model is adequately accurate.

\section{B. System identification and controller parameters design}

Prior to commencing the control design, an identification of the system dynamics within the bandwidth of interest (10 $\mathrm{Hz}-20 \mathrm{kHz}$ ) is first carried out. The frequency response data is recorded using bandlimited white noise as input and a corresponding $z$-domain model is identified. The identified model fully captures the linear dynamics of the nanopositioner axis within the bandwidth of interest as shown in Fig. 5. The resulting $z$-domain transfer-function model is given by:

$$
\begin{aligned}
G(z)=z^{-1} \frac{-1.3906 \mathrm{e}^{-4}\left(z^{2}+2.1708 z+2.196\right)}{\left(z^{2}-0.2944 z+0.2061\right)} \\
\times \frac{\left(z^{2}+3.0375 z+31.9162\right)(z-11.4914)}{\left(z^{2}+0.3720 z+0.8702\right)(z-0.4428)}
\end{aligned}
$$

The controller parameters of the LP FIR-VFDF based Fractional Repetitive Control, LIFRC, conventional RC and PI control schemes are derived using the identified system model parameters. The causal system inversion $C_{i}(z)$ can be computed using Eq. (18), where $n_{u}$ and $n_{c}$ both have a value of 5, and $r=1$. Consequently, the order of the phase compensator is 6 . Simulation results from the step response are used to select the gains for $C_{f}(z)$, which are maximized prior to the occurrence of any significant vibrations. These gains are further tuned during real-time experiments. The proportional and integral gains are finally selected as 0.1 and 6000 , respectively. The non-causal term in the low-pass filter $q(z)$ and $F(z, d)$ can be compensated with a delay term $z^{-\tilde{N}+m}$ in real time control. It is worth of mentioning that, for the pure PI control, the plugged-in RC module is non-existent. To evaluate the four control schemes, their sensitivity functions are compared in Fig. 6, for a specific tracking frequency of $1100 \mathrm{~Hz}$, where the number of the delay points is 45.45 . For the conventional $\mathrm{RC}$ control, the number of the delay points is rounded to 45 . It is found that all the three RC schemes result in significantly lower errors when compared to the baseline scheme with PI tracking controller alone. However, for the conventional RC, due to the non-integer (fractional) ratio between the sampling frequency and the trajectory frequency, the attenuation of the 


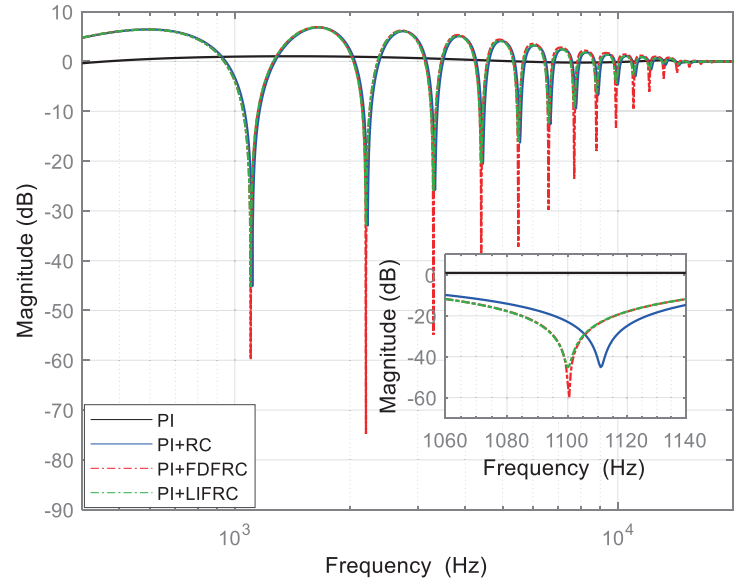

Fig. 6. The sensitivity functions under different control schemes for the tracking frequency of $1100 \mathrm{~Hz}$ (where FDFRC denotes the LP FIR-VFDF based Fractional Repetitive control).

TABLE I

THE CONTROL PARAMETERS FOR DIFFERENT CONTROL SCHEMES (WHERE FDFRC DENOTES THE LP FIR-VFDF BASED FRACTIONAL REPETITIVE CONTROL).

\begin{tabular}{|c|c|c|c|c|c|c|}
\hline Schemes & $k_{p}$ & $k_{i}$ & $k_{c}$ & $\begin{array}{l}\text { Fractional } \\
\text { delay filter }\end{array}$ & $\begin{array}{c}\text { Low-pass } \\
\text { filter }\end{array}$ & $\begin{array}{c}\text { Number of } \\
\text { delay points }\end{array}$ \\
\hline PI & \multirow{4}{*}{0.1} & \multirow{4}{*}{6000} & \multicolumn{4}{|c|}{ Not applicable } \\
\hline $\mathrm{PI}+\mathrm{RC}$ & & & \multirow{3}{*}{1} & Not applicable & $q(z)$ & $\hat{N}=\operatorname{round}(N)$ \\
\hline PI+LIFRC & & & & $L(z, d)$ & $q(z)$ & $\widehat{N}=$ floor $(N)-1$ \\
\hline PI+FDFRC & & & & \multicolumn{2}{|c|}{$F(z, d)$} & $\tilde{N}=$ floor $(N)$ \\
\hline
\end{tabular}

tracking errors at $1100 \mathrm{~Hz}$ is only $-22 \mathrm{~dB}$ as opposed to -45 $\mathrm{dB}$ for the LIFRC and $-60 \mathrm{~dB}$ for the LP FIR-VFDF based Fractional Repetitive Control, respectively. The same trend is seen at higher harmonics. Therefore, it is expected that the LP FIR-VFDF based Fractional Repetitive Control should deliver superior disturbance rejection and result in highly accurate tracking of high-frequency trajectories irrespective of whether the sampling frequency is an integer multiple of the trajectory frequency.

\section{EXPERIMENTAL STUDIES}

To make a comprehensive study, the comparative experiments are conducted on the nanopositioning stage described in Sec. III-A for frequencies ranging from $100 \mathrm{~Hz}$ to 1587.3 $\mathrm{Hz}$, resulting in rounding errors varying between 0 and 0.5 . Low-frequency $(100 \mathrm{~Hz}, 150 \mathrm{~Hz}$ and $200 \mathrm{~Hz})$ triangular trajectories are selected to demonstrate that the proposed scheme possesses good low-frequency tracking performance. A number of high-frequency triangular trajectories with fundamental frequency ranging from $1000 \mathrm{~Hz}$ to $1587.3 \mathrm{~Hz}$ are also tested to showcase the significantly improved high-frequency tracking performance afforded by the proposed LP FIR-VFDF based Fractional Repetitive Control. All triangular trajectories provide a displacement of $2.5 \mu \mathrm{m}$. The control parameters (as shown in Tab. I) are kept constant for all the frequencies.

To evaluate the tracking performance in the steady state, two performance metrics are employed: (i) maximum tracking errors, defined as

$$
e_{m}=\frac{\max _{t \in\left(0,10 T_{r}\right]}\left|y_{d}(t)-y_{a}(t)\right|}{\max \left[y_{d}(t)\right]-\min \left[y_{d}(t)\right]} \times 100 \%
$$

(ii) root-mean-square tracking errors, defined as

$$
e_{r m s}=\frac{\sqrt{\frac{1}{10 T_{r}}} \sum_{t \in\left(0,10 T_{r}\right]}\left[y_{d}(t)-y_{a}(t)\right]^{2}}{\max \left[y_{d}(t)\right]-\min \left[y_{d}(t)\right]} \times 100 \%
$$

where $T_{r}$ is the period of the desired trajectory. The results for these metrics under different control schemes for different tracking frequencies are summarized in Tab. II.

As expected, the baseline PI scheme results in very significant tracking errors even at low frequencies. This is due to the broadband nature of the triangular trajectories (infinite odd harmonics of the fundamental frequency) and the relatively narrow tracking bandwidth afforded by the PI controller alone. Note that $N$ in the second column of the Table II, is the number of delay (sample) points in theory with $N=f_{s} / f_{d}$. Consequently, where $N$ is an integer, the PI+LIFRC as well as the PI+ LP FIR-VFDF based Fractional Repetitive Control (PI+FDFRC) schemes are not necessarily designed while the PI+RC scheme significantly outperforms the PI-only control scheme. Where $N$ is not an integer (fractional delay), the PI+LIFRC delivers superior performance to both the PI as well as the PI+RC schemes. However, in all such fractional delay instances, the newly proposed PI+FDFRC scheme outperforms the traditional PI+LIFRC scheme by quite a margin. This superior performance of the PI+FDFRC scheme is consistent for all the frequencies (including the high-frequencies) tested herewith.

In order to better illustrate the tracking performance achieved by the different control schemes, time-domain tracking results were recorded for the high-frequency $1587.3 \mathrm{~Hz}$ $(N=31.5)$ and $1538.5 \mathrm{~Hz}(N=32.5)$ triangular trajectories, as shown Fig. 7(A) and Fig. 7(B), respectively. In these two particular cases, the rounding errors are both 0.5 . It can be observed from Figs. 7(A-c) and 7(B-c) that, for PI+RC scheme, there exist significant steady-state tracking errors, which degrade the tracking performance greatly. With the PI+LIFRC and PI+FDFRC schemes, these tracking errors are reduced significantly. Moreover, the proposed PI+FDFRC scheme shows a significant reduction in tracking errors when compared with those using the traditional PI+LIFRC scheme. More importantly, the PI+FDFRC scheme reaches steadystate as quickly as the basic PI+RC scheme. These results validate the effectiveness of the proposed PI+FDFRC scheme, in terms of the tracking precision and convergence speed. As a consequence, the proposed PI+FDFRC scheme emerges as a strong contender for the adopted control scheme in high-speed nanopositioning applications.

\section{CONClusion}

This paper proposes the LP FIR-VFDF to enhance the performance of the conventional repetitive control scheme during high-speed nano-scale scanning operations bypassing the restriction of having the sampling frequency be an integer mul- 
TABLE II

THE TRACKING ERRORS UNDER DIFFERENT CONTROL SCHEMES FOR DIFFERENT TRACKING FREQUENCIES (WHERE FDFRC DENOTES THE LP FIR-VFDF BASED FRACTIONAL REPETITIVE CONTROL).

\begin{tabular}{c|c|c|c|c|c|c|c|c|c}
\hline \hline $\begin{array}{c}\text { Frequency } \\
(\mathrm{Hz})\end{array}$ & \multirow{2}{*}{$N$} & \multicolumn{2}{|c|}{ PI (\%) } & \multicolumn{2}{c|}{ PI+RC (\%) } & \multicolumn{2}{|c|}{ II+LIFRC (\%) } & \multicolumn{2}{|c}{ PI+FDFRC (\%) } \\
\cline { 3 - 10 } & $e_{m}$ & $\mathrm{e}_{\mathrm{r} m s}$ & $e_{m}$ & $\mathrm{e}_{\mathrm{r} m s}$ & $e_{m}$ & $e_{r m s}$ & $e_{m}$ & $e_{r m s}$ \\
\hline 100 & 500 & 14.343 & 11.775 & 0.182 & 0.048 & - & - & - & - \\
150 & 333.33 & 20.500 & 16.850 & 0.528 & 0.139 & 0.206 & 0.052 & 0.212 & 0.051 \\
200 & 250 & 26.380 & 21.113 & 0.160 & 0.051 & - & - & - & - \\
1000 & 50 & 52.636 & 31.946 & 1.549 & 0.390 & - & - & - & - \\
1100 & 45.45 & 52.783 & 31.918 & 3.594 & 2.241 & 1.669 & 0.444 & 0.595 & 0.226 \\
1200 & 41.67 & 52.774 & 31.967 & 3.140 & 1.845 & 1.973 & 0.522 & 0.918 & 0.350 \\
1300 & 38.46 & 52.735 & 31.929 & 4.438 & 2.709 & 2.251 & 0.582 & 0.945 & 0.328 \\
1400 & 35.71 & 52.682 & 31.801 & 3.540 & 1.999 & 2.693 & 0.722 & 1.287 & 0.510 \\
1500 & 33.33 & 52.472 & 31.688 & 4.243 & 2.375 & 2.974 & 0.789 & 1.610 & 0.536 \\
1538.5 & 32.5 & 52.317 & 31.755 & 5.627 & 3.585 & 3.031 & 0.815 & 1.444 & 0.495 \\
1562.5 & 32 & 52.347 & 31.554 & 3.031 & 0.807 & - & - & - & - \\
1587.3 & 31.5 & 52.305 & 31.309 & 5.581 & 3.754 & 3.153 & 0.847 & 1.536 & 0.505 \\
\hline \hline
\end{tabular}
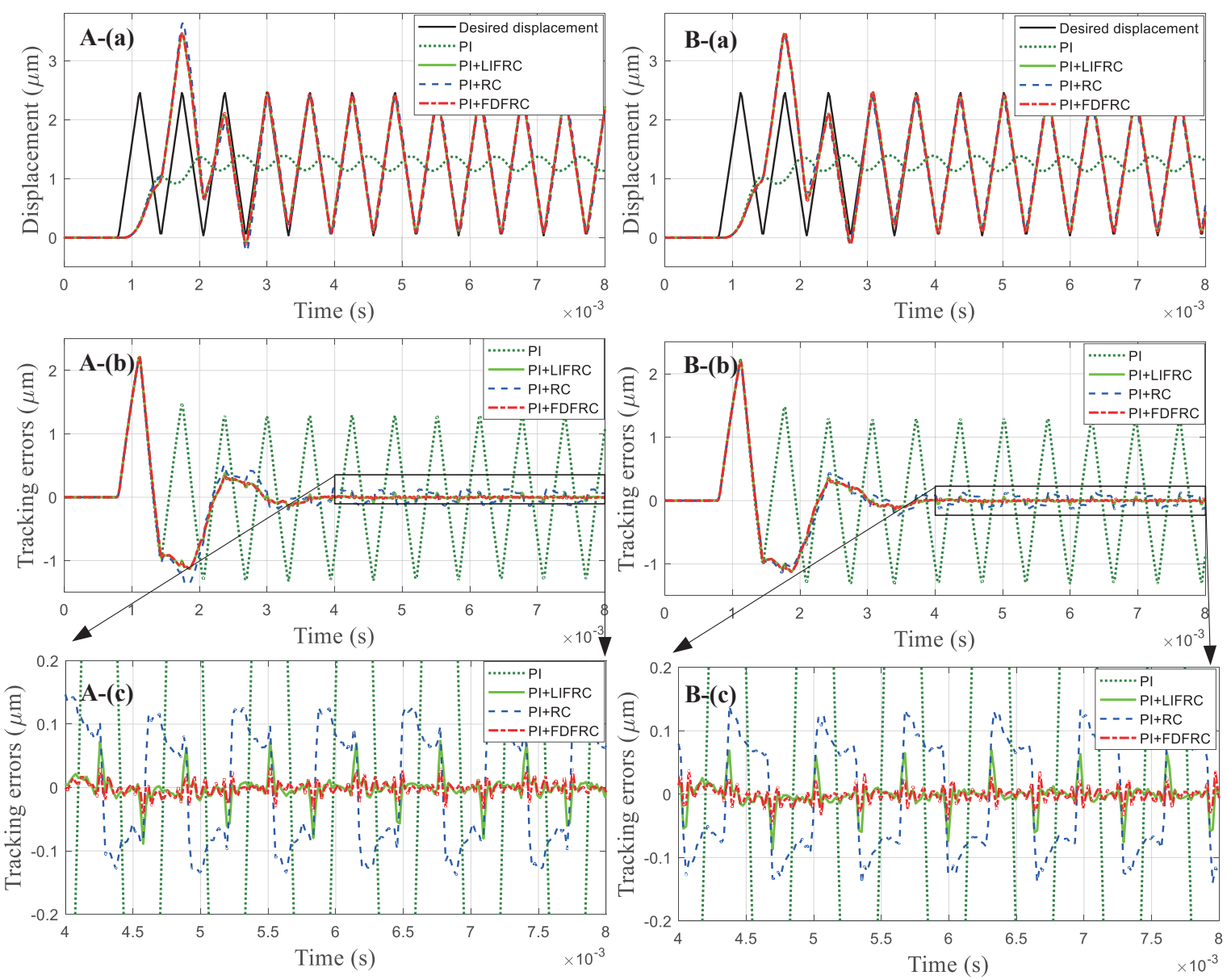

Fig. 7. The tracking results under different control schemes for different tracking frequencies: $(\mathrm{A}) 1587.3 \mathrm{~Hz}(N=31.5)$; $(\mathrm{B}) 1538.5 \mathrm{~Hz}(N=32.5)$; (a) The overall tracking results; (b) The tracking errors; (c) The enlarged tracking errors in steady state.

tiple of the trajectory frequency. The filter works by combining approximation of the fractional delay and low-pass behavior together, as an alternative to the commonly used low-pass filter cascading with the fractional delay approximation. The weighted-least-square method is employed to optimally design the variable fractional delay filters, which minimizes the approximation error within the interested bandwidth. The comparative tracking experiments with the conventional $\mathrm{PI}+\mathrm{RC}$, PI+LIFRC, and the pure PI control schemes are conducted on a custom-built nanopositioning stage for frequencies ranging 
from the low-frequency of $150 \mathrm{~Hz}$ to the high-frequency of 1587.3 Hz. The experimental results show that the proposed LP FIR-VFDF based Fractional Repetitive Control scheme outperforms the conventional LIFRC scheme of the same order in terms of tracking performance. Triangular trajectories with a fundamental frequency up to $1587.3 \mathrm{~Hz}$ were accurately tracked by implementing the proposed LP FIR-VFDF based Fractional Repetitive Control with positioning errors as low as $0.505 \%$ - clearly highlighting the proposed schemes efficacy at delivering high-speed nanopositioning.

\section{Appendix A. Proof of The EXISTENCE of}

$$
\left(P^{T} P+Q^{T} Q\right)^{-1}
$$

Expanding Eq. (11), we could have

$$
\begin{gathered}
P=\left[\begin{array}{cccc}
W^{\frac{1}{2}}\left(\omega_{0}\right) & & & \\
& & & \\
& W^{\frac{1}{2}\left(\omega_{1}\right)} & & \\
& \ddots & \\
& & W^{\frac{1}{2}}\left(\omega_{S_{\omega}}\right)
\end{array}\right] \times \\
{\left[\begin{array}{cccc} 
& & \\
\cos \left(N_{1} \omega_{0}\right) & \cos \left[\left(N_{1}+1\right) \omega_{0}\right] & \cdots & \cos \left(N_{2} \omega_{0}\right) \\
\cos \left(N_{1} \omega_{1}\right) & \cos \left[\left(N_{1}+1\right) \omega_{1}\right] & \cdots & \cos \left(N_{2} \omega_{1}\right) \\
\vdots & & \ddots & \vdots \\
\cos \left(N_{1} \omega_{S_{\omega}}\right) & \cos \left[\left(N_{1}+1\right) \omega_{S_{\omega}}\right] & \cdots & \cos \left(N_{2} \omega_{S_{\omega}}\right)
\end{array}\right]}
\end{gathered}
$$

and hence $P^{T} P$ can be expressed as $P_{c}^{T} W P_{c}$, with

$$
P_{c}=\left[\begin{array}{cccc}
\cos \left(N_{1} \omega_{0}\right) & \cos \left[\left(N_{1}+1\right) \omega_{0}\right] & \cdots & \cos \left(N_{2} \omega_{0}\right) \\
\cos \left(N_{1} \omega_{1}\right) & \cos \left[\left(N_{1}+1\right) \omega_{1}\right] & \cdots & \cos \left(N_{2} \omega_{1}\right) \\
\vdots & \vdots & \ddots & \vdots \\
\cos \left(N_{1} \omega_{S_{\omega}}\right) & \cos \left[\left(N_{1}+1\right) \omega_{S_{\omega}}\right] & \cdots & \cos \left(N_{2} \omega_{S_{\omega}}\right)
\end{array}\right]
$$

and

$$
W=\left[\begin{array}{llll}
W\left(\omega_{0}\right) & & & \\
& & & \\
& W\left(\omega_{1}\right) & & \\
& & \ddots & \\
& & & \\
& & W\left(\omega_{S_{\omega}}\right)
\end{array}\right]
$$

Similarly, from Eq. (12), $Q^{T} Q$ can be described by $Q_{c}{ }^{T} W Q_{c}$, where

$$
Q_{c}=\left[\begin{array}{cccc}
\sin \left(N_{1} \omega_{0}\right) & \sin \left[\left(N_{1}+1\right) \omega_{0}\right] & \ldots & \sin \left(N_{2} \omega_{0}\right) \\
\sin \left(N_{1} \omega_{1}\right) & \sin \left[\left(N_{1}+1\right) \omega_{1}\right] & \ldots & \sin \left(N_{2} \omega_{1}\right) \\
\vdots & \vdots & \ddots & \vdots \\
\sin \left(N_{1} \omega_{S_{\omega}}\right) & \sin \left[\left(N_{1}+1\right) \omega_{S_{\omega}}\right] & \ldots & \sin \left(N_{2} \omega_{S_{\omega}}\right)
\end{array}\right]
$$

Let $X \in R^{\left(N_{2}-N_{1}+1\right) \times 1}$ be an arbitrary vector. When the relationship $X^{T}\left(P^{T} P+Q^{T} Q\right) X>0$ is satisfied, $P^{T} P+Q^{T} Q$ is clearly positive definite. Denote $X^{T} P_{c}^{T}=\left[\begin{array}{llll}C_{0} & C_{1} & \ldots & C_{S_{\omega}}\end{array}\right]$, and $X^{T} Q_{c}{ }^{T}=$ $\left[\begin{array}{llll}S_{0} & S_{1} & \ldots & S_{S_{\omega}}\end{array}\right]$, then:

$$
\begin{aligned}
& X^{T}\left(P^{T} P+Q^{T} Q\right) X=X^{T}\left(P_{c}^{T} W P_{c}+Q_{c}{ }^{T} W Q_{c}\right) X \\
& =W\left(\omega_{0}\right)\left(C_{0}{ }^{2}+{S_{0}}^{2}\right)+W\left(\omega_{1}\right)\left(C_{1}{ }^{2}+S_{1}{ }^{2}\right)+\cdots \\
& \quad+W\left(\omega_{s_{\omega}}\right)\left(C_{s_{\omega}}{ }^{2}+S_{s_{\omega}}{ }^{2}\right)
\end{aligned}
$$

The condition $W\left(\omega_{i}\right)>0$ is always satisfied. Following $\left(C_{i}{ }^{2}+S_{i}{ }^{2}\right)>0$, the proof can be completed. In this sense, the problem of positive definite of $P^{T} P+Q^{T} Q$ resolve into a problem of positive definite of $P_{c}^{T} P_{c}+Q_{c}{ }^{T} Q_{c}$. Substituting Eqs. (A.2) and (A.4) into $P_{c}^{T} P_{c}+Q_{c}^{T} Q_{c}$,

$$
\begin{aligned}
& P_{c}^{T} P_{c}+Q_{c}{ }^{T} Q_{c}= \\
& {\left[\begin{array}{cccc}
S_{\omega}+1 & \sum_{i=0}^{S_{\omega}} \cos \omega_{i} & \cdots & \sum_{i=0}^{S_{\omega}} \cos n \omega_{i} \\
\sum_{i=0}^{S_{\omega}} \cos \omega_{i} & S_{\omega}+1 & \cdots & \sum_{i=0}^{S_{\omega}} \cos (n-1) \omega_{i} \\
\vdots & \vdots & \ddots & \vdots \\
\sum_{i=0}^{S_{\omega}} \cos n \omega_{i} & \sum_{i=0}^{S_{\omega}} \cos (n-1) \omega_{i} & \cdots & S_{\omega}+1
\end{array}\right]}
\end{aligned}
$$

As $\omega_{i}$ is distributed uniformly in $[0, \pi], \sum_{i=0}^{S_{\omega}} \cos n \omega_{i}=0$. Therefore, $P_{c}^{T} P_{c}+Q_{c}{ }^{T} Q_{c}$ is positive definite, and so is $P^{T} P+Q^{T} Q$. The proof is thus completed.

Appendix B. Proof of the existence of $\left(D^{T} D\right)^{-1}$ In view of the definition,

$$
D=\left[d_{j}^{k}\right]=\left[\begin{array}{cccc}
d_{0}^{0} & d_{0}^{1} & \cdots & d_{0}^{k} \\
d_{1}^{0} & d_{1}^{1} & \cdots & d_{1}^{k} \\
\vdots & \vdots & & \vdots \\
d_{S_{d}}^{0} & d_{S_{d}}^{1} & \cdots & d_{S_{d}}^{k}
\end{array}\right]
$$


where $d_{j} \neq d_{i}(i \neq j)$. Thus, $\operatorname{rank}(D)=\min \left(S_{d}+1, K+1\right)$. As $D^{T} D$ has the dimension of $K \times K$, the term $D^{T} D$ is full rank, when $K<S_{d}$. The proof is thus completed.

\section{REFERENCES}

[1] G. Binning, C. F. Quate, and C. Gerber, "Atomic force microscope," Phys. Rev. Lett., no. 56, p. 9, 1986.

[2] T. Watanabe-Nakayama, M. Itami, N. Kodera, T. Ando, and H. Konno, "High-speed atomic force microscopy reveals strongly polarized movement of clostridial collagenase along collagen fibrils," Sci. Rep., vol. 6, no. 1, p. 28975, Sep. 2016.

[3] M. Shibata, H. Nishimasu, N. Kodera et al., "Real-space and realtime dynamics of CRISPR-Cas9 visualized by high-speed atomic force microscopy," Nat. Commun., vol. 8, no. 1, p. 1430, Dec. 2017.

[4] S. Devasia, E. Eleftheriou, and S. O. R. Moheimani, "A Survey of Control Issues in Nanopositioning," IEEE Trans. Control Syst. Technol., vol. 15 , no. 5, pp. 802-823, Sep. 2007

[5] G.-Y. Gu, L.-M. Zhu, C.-Y. Su, and H. Ding, "Modeling and Control of Piezo-Actuated Nanopositioning Stages: A Survey, " IEEE Trans. Autom. Sci. Eng., vol. 13, no. 1, p. 20, 2016.

[6] M. S. Rana, H. R. Pota, and I. R. Petersen, "Improvement in the Imaging Performance of Atomic Force Microscopy: A Survey, " IEEE Trans. Autom. Sci. Eng., vol. 14, no. 2, pp. 1265-1285, Apr. 2017.

[7] G. M. Clayton, S. Tien, K. K. Leang, Q. Zou, and S. Devasia, "A Review of Feedforward Control Approaches in Nanopositioning for High-Speed SPM, ” J. Dyn. Syst. Meas. Control, vol. 131, no. 6, p. 061101, 2009.

[8] G. Schitter, P. J. Thurner, and P. K. Hansma, "Design and input-shaping control of a novel scanner for high-speed atomic force microscopy," Mechatronics, vol. 18, no. 5-6, pp. 282-288, Jun. 2008.

[9] A. J. Fleming and A. G. Wills, "Optimal Periodic Trajectories for BandLimited Systems, " IEEE Trans. Control Syst. Technol., vol. 17, no. 3, pp. 552-562, May 2009.

[10] A. Bazaei, Y. K. Yong, and S. O. R. Moheimani, "Combining Spiral Scanning and Internal Model Control for Sequential AFM Imaging at Video Rate," IEEE/ASME Trans. Mechatron., vol. 22, no. 1, pp. 371380, Feb. 2017.

[11] M. S. Rana, H. R. Pota, and I. R. Petersen, "Performance of Sinusoidal Scanning With MPC in AFM Imaging, "IEEE/ASME Trans. Mechatron., vol. 20, no. 1, pp. 73-83, Feb. 2015.

[12] K. K. Leang and S. Devasia, "Feedback-Linearized Inverse Feedforward for Creep, Hysteresis, and Vibration Compensation in AFM Piezoactuators, "IEEE Trans. Control Syst. Technol., vol. 15, no. 5, p. 9, 2007.

[13] S. S. Aphale, B. Bhikkaji, and S. O. R. Moheimani, "Minimizing Scanning Errors in Piezoelectric Stack-Actuated Nanopositioning Platforms, "IEEE Trans. Nanotechnol., vol. 7, no. 1, pp. 79-90, Jan. 2008.

[14] L. Li, C.-X. Li, G. Gu, and L.-M. Zhu, "Positive acceleration, velocity and position feedback based damping control approach for piezoactuated nanopositioning stages, " Mechatronics, vol. 47, pp. 97-104, Nov. 2017.

[15] S. K. Das, H. R. Pota, and I. R. Petersen, "A MIMO Double Resonant Controller Design for Nanopositioners, "IEEE Trans. Nanotechnol., vol. 14, no. 2, pp. 224-237, Mar. 2015.

[16] A. J. Fleming, S. S. Aphale, and S. O. R. Moheimani, "A New Method for Robust Damping and Tracking Control of Scanning Probe Microscope Positioning Stages, "IEEE Trans. Nanotechnol., vol. 9, no. 4, pp. 438-448, Jul. 2010.

[17] S. S. Aphale, A. Ferreira, and S. O. R. Moheimani, "A robust loopshaping approach to fast and accurate nanopositioning," Sens. Actuators Phys., vol. 204, pp. 88-96, Dec. 2013.

[18] S. Salapaka, A. Sebastian, J. P. Cleveland, and M. V. Salapaka, "High bandwidth nano-positioner: A robust control approach," Rev. Sci. Instrum., vol. 73, no. 9, pp. 3232-3241, Sep. 2002.

[19] A. San-Millan, V. Feliu-Batlle, and S. S. Aphale, "Fractional order implementation of Integral Resonant Control - A nanopositioning application," ISA Trans., vol. 82, pp. 223-231, Nov. 2018.

[20] C.-X. Li, Y. Ding, G.-Y. Gu, and L.-M. Zhu, "Damping Control of PiezoActuated Nanopositioning Stages With Recursive Delayed Position Feedback, "IEEE/ASME Trans. Mechatron., vol. 22, no. 2, pp. 855864, Apr. 2017.

[21] Y. Jian, D. Huang, J. Liu, and D. Min, "High-Precision Tracking of Piezoelectric Actuator Using Iterative Learning Control and Direct Inverse Compensation of Hysteresis, "IEEE Trans. Ind. Electron., vol. 66, no. 1, pp. 368-377, Jan. 2019.
[22] Y. Wu, J. Shi, C. Su, and Q. Zou, "A control approach to crosscoupling compensation of piezotube scanners in tapping-mode atomic force microscope imaging, " Rev. Sci. Instrum., vol. 80, no. 4, p. 043709, Apr. 2009.

[23] S. Tien, Qingze Zou, and S. Devasia, "Iterative control of dynamicscoupling-caused errors in piezoscanners during high-speed AFM operation," IEEE Trans. Contr. Syst. Technol., vol. 13, no. 6, pp. 921-931, Nov. 2005.

[24] C.-Y. Lin and P.-Y. Chen, "Precision tracking control of a biaxial piezo stage using repetitive control and double-feedforward compensation," Mechatronics, vol. 21, no. 1, pp. 239-249, Feb. 2011.

[25] K.-S. Kim and Q. Zou, "A Modeling-Free Inversion-Based Iterative Feedforward Control for Precision Output Tracking of Linear TimeInvariant Systems, "IEEE/ASME Trans. Mechatron., vol. 18, no. 6, pp. 1767-1777, Dec. 2013.

[26] R. de Rozario and T. Oomen, "Data-driven iterative inversion-based control: Achieving robustness through nonlinear learning, " Automatica, vol. 107, pp. 342-352, Sep. 2019.

[27] M. Zhang and Z. Lu, "Circulating Harmonic Current Elimination of a CPS-PWM Based Modular Multilevel Converter with Plug-In Repetitive Controller," IEEE Trans. Power Electron., vol. 29, no. 4, pp. 2083-2097, Apr. 2014.

[28] Y. Shan and K. K. Leang, "Accounting for hysteresis in repetitive control design: Nanopositioning example," Automatica, vol. 48, no. 8, pp. 17511758, Aug. 2012

[29] C.-X. Li and L.-M. Zhu, "High-Speed Tracking of a Nanopositioning Stage Using Modified Repetitive Control, "IEEE Trans. Autom. Sci. Eng., vol. 14, no. 3, p. 11, 2017.

[30] Y. Shan and K. K. Leang, "Dual-stage repetitive control with PrandtlCIshlinskii hysteresis inversion for piezo-based nanopositioning," Mechatronics, vol. 22, no. 3, pp. 271-281, Apr. 2012.

[31] L. Li, G. Gu, and L. Zhu, "Fractional repetitive control of nanopositioning stages for tracking high-frequency periodic inputs with nonsynchronized sampling, ” Rev. Sci. Instrum., vol. 90, no. 5, p. 055108, May 2019.

[32] D. Wang and X. Chen, "A multirate fractional-order repetitive control for laser-based additive manufacturing, " Control Eng. Pract., vol. 77, pp. 41-51, Aug. 2018.

[33] Z. Liu, B. Zhang, and K. Zhou, "Universal Fractional-Order Design of Linear Phase Lead Compensation Multirate Repetitive Control for PWM Inverters, " IEEE Trans. Ind. Electron., vol. 64, no. 9, pp. 7132-7140, Sep. 2017.

[34] P. Cui, Q. Wang, S. Li, and Q. Gao, "Combined FIR and FractionalOrder Repetitive Control for Harmonic Current Suppression of Magnetically Suspended Rotor System, "IEEE Trans. Ind. Electron., vol. 64, no. 6, pp. 4828-4835, Jun. 2017

[35] S.-C. Pei, Y.-D. Huang, S.-H. Lin, and J.-J. Shyu, "Design of variable comb filter using FIR variable fractional delay element," Signal Process., p. 13, 2012.

[36] T.-B. Deng and Y. Lian, "Weighted-Least-Squares Design of Variable Fractional-Delay FIR Filters Using Coefficient Symmetry," IEEE Trans. Signal Process., vol. 54, no. 8, pp. 3023-3038, Aug. 2006.

[37] M. Tomizuka, "Zero Phase Error Tracking Algorithm for Digital Control, "Journal of Dynamic Systems, Measurement, and Control, vol. 109, no. 1, p. 65, 1987.

[38] Q. Zhao and Y. Ye, "Fractional Phase Lead Compensation RC for an Inverter: Analysis, Design, and Verification, " IEEE Trans. Ind. Electron., vol. 64, no. 4, pp. 3127-3136, Apr. 2017.

[39] C.-X. Li, G.-Y. Gu, M.-J. Yang, and L.-M. Zhu, "Design, analysis and testing of a parallel-kinematic high-bandwidth XY nanopositioning stage, "Rev. Sci. Instrum., vol. 84, no. 12, p. 125111, Dec. 2013.

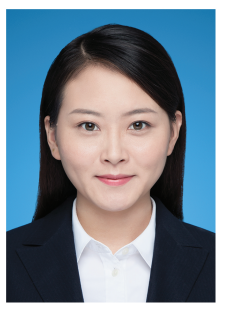

Linlin Li received the B.E. degree (with honors) in mechanical design, manufacturing, and automation from Shandong University, Jinan, China, in 2014. She is currently working toward the Ph.D. degree in mechanical engineering at Shanghai Jiao Tong University, Shanghai, China.

Her research interests include mechatronics, modeling and control of high-bandwidth nanopositioning stages, and atomic force microscopes. 


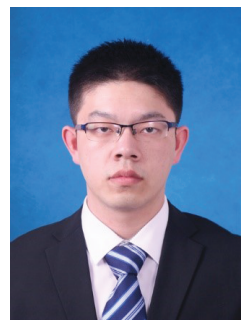

Zaozao Chen received the B.E. degree in mechanical engineering from Shanghai Jiao Tong University, Shanghai, China, in 2017, where he is currently working toward the Ph.D. degree in mechanical engineering. His research interests include signal processing, error modeling, detecting and compensation of multi-axis CNC machine tool.

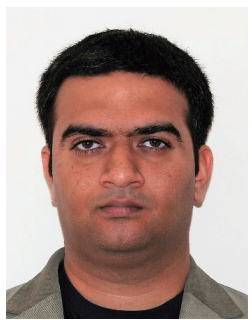

Sumeet S. Aphale (Ph.D., CEng, SMIEEE, MIET) is a Senior Lecturer and a Chartered Engineer at the School of Engineering, University of Aberdeen, UK. His formal education includes Bachelors (University of Pune, India 1999), Masters (University of Wyoming, USA 2003) and PhD (University of Wyoming, USA, 2005) degrees, all in Electrical Engineering with a focus on Robotics and Control. He has held post-doctoral research positions at the ARC Centre of Excellence for Complex Dynamic Systems and Control, University of Newcastle (2006-08) and at the Centre for Applied Dynamics Research, University of Aberdeen (200809). His research interests lie in the broad area of mechatronics, applied control and robotics. He has published over 70 papers in peer-reviewed journals and conferences focussing on control of nanopositioning systems, flexible robots, parallel robots, vibration control, drill-string dynamics, biomedical device designs and fibre-optic sensors. He is an Associate Editor for the IEEE Control Systems Societys Conference Editorial Board. He is also serves as an Associate Editor for Shock and Vibration as well as for Frontiers of Mechanical Engineering (Mechatronics Section).

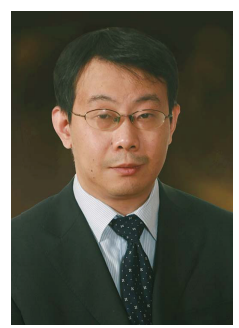

LiMin Zhu received the B.E. degree (with honors) and the $\mathrm{Ph} . \mathrm{D}$. degree in mechanical engineering from Southeast University in 1994 and 1999, respectively. From Nov. 1999 to Jan. 2002, he worked as a postdoctoral fellow in Huazhong University of Science and Technology. Since March 2002, he has been with Shanghai Jiao Tong University, where he is currently the Cheung Kong Chair Professor, head of the Department of Mechanical Engineering and vice director of the State Key Laboratory of Mechanical System and Vibration. He has held the visitorship in Monash University (from Sep. 1997 to May 1998) and The City University of Hong Kong (from Dec. 2000 to Mar. 2001). His research interests include (1) Multi-axis CNC machining technology and machine tool, (2) coordinate metrology and measurement, and (3) control, sensing and instrumentation for micro/nano manufacturing. He has published 1 monograph and more than 170 international journal papers.

Prof. Zhu was the recipient of the National Science Fund for Distinguished Young Scholars in 2013 and selected into the National High-level Personnel of Special Support Program in 2016. He has been an Associate Editor for the IEEE Transactions on Automation Science and Engineering. He is now a Technical Editor for the IEEE/ASME Transactions on Mechatronics, and Editorial Board Members of the Proceedings of the Institution of Mechanical Engineer (IMechE), Part B: Journal of Engineering Manufacture, and Chinese Journal of Mechanical Engineering. 